\title{
Sound wave acceleration in granular materials
}

\section{O Mouraille ${ }^{a, *}$, W A Mulder ${ }^{b} \&$ S Luding ${ }^{a}$}

${ }^{a}$ Particle Technology, Nanostructured Materials, DelftChemTech, TUDelft, Julianalaan 136, 2628 BL Delft, Netherlands

${ }^{b}$ Applied Geophysics and Petrophysics, Civil Engineering and Geotechnology, Delft, Netherlands

E-mail: * o.mouraille@tnw.tudelft.nl

\begin{abstract}
The goal of this study is a better understanding of the numerous sound propagation mechanisms in granular materials. In a static, regular (crystal), 3D packing, a small perturbation is created on one side and examined during its propagation through frictionless and frictional packings. The perturbation can be applied in longitudinal and shear direction in order to excite different modes of information propagation, including rotational modes as well. Wave speed and dispersion relation derived from simulation data are compared to those given by a theoretical approach based on a micro-macro transition. The detailed analysis of the wave velocity reveals an interesting acceleration close to the source. Finally a step towards real packings is made by introducing either friction or a tiny (but decisive) polydispersity in the particle size.
\end{abstract}




\section{Introduction}

The mechanisms of wave propagation through a given, possibly random, disordered material are strongly related to the properties of this material. These include the stiffness and the structure, where anisotropy comes into play, but also phenomena like dissipation or friction at the "micro-" or contact-level, see Refs. [1, 2, 3]. Due to friction, particles rotate and hence the rotational degrees of freedom can also play an important role. Such "micro" phenomena can be modeled with a discrete approach [4, 6], but applications such as oil field exploration and the treatment of seismic data in general, involve larger ("macro") scales and hence do not allow us to view the material in such detail. A continuum description [7] is therefore needed and this leads to the issue of a "micro-macro" transition for information propagation in granular materials.

In the following, section 2 describes the discrete MD (Molecular Dynamics) model used, in particular, the implementation of contacts and friction, and the granular packing structure as well. Section 3 details the influence of dissipation and friction and the difference between modes of agitation and propagation (compressive/shear). The wave speed is analysed and compared to the continuum theory approach. Also the dispersion relation is extracted from the data and compared to theoretical predictions. In the last paragraph the influence of small perturbations in the ordered structure of the packing (applying a tiny size distribution to the particles) on the wave propagation is examined.

\section{DESCRIPTION OF THE MODEL}

\subsection{Discrete Particle Model}

The elementary units of granular materials are mesoscopic grains, which deform under the stress developing at their contacts. Since realistic modeling of the internal deformation of the particles is much too complicated, we relate the normal interaction force to the overlap $\delta$ of two spherical particles. If the sum of all forces, $\boldsymbol{f}_{i}$, acting on particle $i$, either from other particles, from boundaries or from external forces, is known, the problem is reduced to the integration of Newton's equations of motion for the translational and rotational degrees of freedom:

$$
m_{i} \frac{\mathrm{d}^{2}}{\mathrm{~d} t^{2}} \boldsymbol{r}_{i}=\boldsymbol{f}_{i}, \quad \text { and } \quad I_{i} \frac{\mathrm{d}^{2}}{\mathrm{~d} t^{2}} \boldsymbol{\varphi}_{i}=\boldsymbol{t}_{i}
$$

with the mass $m_{i}$ of particle $i$, its position $\boldsymbol{r}_{i}$, its moment of inertia $I_{i}$, its angular velocity $\boldsymbol{\omega}_{i}=\mathrm{d} \boldsymbol{\varphi}_{i} / \mathrm{d} t$ and the total torque $\boldsymbol{t}_{i}$. Note that the above equation is only valid for isotropic bodies, like spheres as used in this study. The force acting on particle $i$ from particle $j$ can be decomposed into a normal and a tangential part.

\section{Linear normal contact law:}

If elasticity and dissipation are desired, the simplest force law in normal direction, $\boldsymbol{n}=\left(\boldsymbol{r}_{i}-\boldsymbol{r}_{j}\right) /\left|\boldsymbol{r}_{i}-\boldsymbol{r}_{j}\right|$, is a linear spring and a linear dashpot $f_{i}^{n}=k \delta+\gamma_{0} \dot{\delta}$, with spring constant $k$ and some damping coefficient $\gamma_{0}$. The half-period of a vibration around the 
equilibrium position leads to a typical response time $t_{c}=\pi / \omega$, with the eigenfrequency of the contact, $\omega=\sqrt{\left(k / m_{i j}\right)-\eta_{0}^{2}}$, the reduced mass, $m_{i j}=m_{i} m_{j} /\left(m_{i}+m_{j}\right)$, and the rescaled damping coefficient, $\eta_{0}=\gamma_{0} /\left(2 m_{i j}\right)$. The energy dissipation during a collision, as caused by the dashpot, leads to a restitution coefficient $r=-v_{n}^{\prime} / v_{n}=\exp \left(-\eta_{0} t_{c}\right)$, where the prime denotes the normal velocity after a collision.

\section{Tangential Contact Model:}

The force in the tangential direction, $\boldsymbol{f}_{t}$ is implemented in the spirit of Ref. [8], where a tangential spring was introduced to account for static friction. For related literature, see Refs. [9, 11, 12]. In the static case, the tangential force is coupled to the normal force via Coulombs law, i.e., $f^{t} \leq \mu^{s} f^{n}$, whereas for the sliding case one has dynamic friction with $f^{t}=\mu^{d} f^{n}$. The dynamic and the static friction coefficients follow generally the relation $\mu^{d} \leq \mu^{s}$. The static case requires an elastic spring, related to the tangential displacement, to allow for a static restoring force, i.e., a non-zero tangential force in static equilibrium due to activated Coulomb friction.

If a contact exists with non-zero normal force, the tangential force is active too, and we project (rotate) the tangential spring, with deformation $\boldsymbol{\xi}$, into the actual tangential plane (this is necessary, since the frame of reference of the contact may have rotated since the last time-step)

$$
\boldsymbol{\xi}=\left|\boldsymbol{\xi}^{\prime}\right| \boldsymbol{t}^{*}, \text { with } \boldsymbol{t}^{*}=\boldsymbol{\xi}^{*} /\left|\boldsymbol{\xi}^{*}\right|, \text { and } \boldsymbol{\xi}^{*}=\boldsymbol{\xi}^{\prime}-\boldsymbol{n}\left(\boldsymbol{n} \cdot \boldsymbol{\xi}^{\prime}\right),
$$

where $\boldsymbol{\xi}^{\prime}$ refers to the tangential spring at the previous iteration, $\boldsymbol{\xi}^{*}$ is the spring projected into the tangential plane, and $\boldsymbol{n}(\boldsymbol{t})$ are the normal (tangential) unit vectors. This action is relevant only for an already existing spring; if the spring is new, the tangential spring-length is zero anyway, however, its evolution is well defined as shown below. The tangential velocity,

$$
\boldsymbol{v}_{t}=\boldsymbol{v}_{i j}-\boldsymbol{n}\left(\boldsymbol{n} \cdot \boldsymbol{v}_{i j}\right),
$$

is computed from the total relative velocity of the surfaces of the two contacting particles,

$$
\boldsymbol{v}_{i j}=\boldsymbol{v}_{i}-\boldsymbol{v}_{j}+a_{i} \boldsymbol{n} \times \boldsymbol{\omega}_{i}+a_{j} \boldsymbol{n} \times \boldsymbol{\omega}_{j} .
$$

Next, we calculate the tangential test-force as the sum of the tangential elastic and viscous forces (in analogy to the normal force model),

$$
\boldsymbol{f}_{\mathrm{o}}^{t}=-k_{t} \boldsymbol{\xi}-\gamma_{t} \boldsymbol{v}_{t}
$$

with the tangential spring stiffness $k_{t}$ and a tangential dissipation parameter $\gamma_{t}$. As long as $\left|\boldsymbol{f}_{\mathrm{o}}^{t}\right| \leq f_{C}^{s}$, with $f_{C}^{s}=\mu^{s} f^{n}$, one has the static friction case (1) and, on the other hand, if $\left|\boldsymbol{f}_{\mathrm{o}}^{t}\right|$ becomes larger than $f_{C}^{s}$, the sliding, dynamic friction case (2) is active with the (possibly lower) Coulomb limit $f_{C}^{d}=\mu^{d} f^{n}$. Sliding case (2) is active as long as, in the next steps, the test force remains $\left|\boldsymbol{f}_{\mathrm{o}}^{t}\right|>f_{C}^{d}$. If the tangential force drops below the dynamic Coulomb limit, $\left|\boldsymbol{f}_{\mathrm{o}}^{t}\right| \leq f_{C}^{d}$, static friction becomes active again, with the (possibly larger) Coulomb limit $f_{C}^{s}$, giving rise to stick-slip behavior. Typically, a contact starts with finite tangential velocity and $\boldsymbol{\xi}=0$; during the first time-steps, the spring is stretched and the velocity decreases. 
In the static case (1), the tangential spring is incremented

$$
\boldsymbol{\xi}^{\prime}=\boldsymbol{\xi}+\boldsymbol{v}_{t} \delta t_{\mathrm{MD}}
$$

with the time step $\delta t_{\mathrm{MD}}$ of the DEM simulation. The new value of $\boldsymbol{\xi}^{\prime}$ is to be used in the next iteration in Eq. (2), along with the tangential force $\boldsymbol{f}^{t}=\boldsymbol{f}_{\mathrm{o}}^{t}$ as defined in Eq. (5).

In the sliding case (2), the tangential spring is adjusted to a length, which is consistent with the Coulomb condition,

$$
\boldsymbol{\xi}^{\prime}=-\frac{1}{k_{t}}\left(f_{C}^{d} \boldsymbol{t}+\gamma_{t} \boldsymbol{v}_{t}\right)
$$

with the tangential unit vector, $\boldsymbol{t}=\boldsymbol{f}_{\mathrm{o}}^{t} /\left|\boldsymbol{f}_{\mathrm{o}}^{t}\right|$, defined by the direction of the force in Eq. (5). Note that this guarantees that the force magnitude does not exceed the Coulomb limit: Inserting $\boldsymbol{\xi}^{\prime}$ into Eq. (5) leads to $\boldsymbol{f}_{\mathrm{o}}^{t} \approx f_{C}^{d} \boldsymbol{t}$, where $\boldsymbol{f}_{\mathrm{o}}^{t}$ and $\boldsymbol{v}_{t}$ are not necessarily parallel to each other in 3D. In short notation the tangential force on particle $i$ reads

$$
\boldsymbol{f}_{i}^{t}=\min \left(f_{C},\left|\boldsymbol{f}_{\mathrm{o}}^{t}\right|\right) \boldsymbol{t}
$$

where $f_{C}$ follows the selection rules described above.

\subsection{Model system}

The configuration considered here is a dense, static packing of grains contained in a cuboid. In the following, monodisperse, structured (crystal) packings are studied, and a few results obtained by introducing a small polydispersity in the particle size of $0.2 \%$, is presented. A more detailed study where both (large) polydispersity and disorder are considered, will be presented elsewhere [14]. The monodisperse structured packing used here is a Face Centered Cubic (FCC) packing: square-layers in the $x$ - $y$-plane are stacked on top of each other (in $z$-direction), such that each layer fits into the holes of the one below, and each second layer is just a $z$-shifted copy of the original. The distance between square layers is $l_{0}=d / \sqrt{2}$ for a particle diameter $d$. In Fig. 1 a part of a packing with 3200 particles is displayed, with 4 times 4 particles in the $x, y$ planes $\left(l_{x}=l_{y}=4 d\right)$ and 200 layers in $z$-direction $\left(l_{z}=200 l_{0}\right.$ - only about 90 layers are shown). Based on a particle-centred square in the first layer, a unit-cell (cuboid) therefore has a volume $V_{u}=\sqrt{2} d^{3}$ and contains 2 particles with volume $2 V_{p}=(\pi / 3) d^{3}$ such that the volume fraction is $\nu=2 V_{p} / V_{u}=\pi /(3 \sqrt{2}) \approx 0.74$. Each particle has four contacts inside each square-layer, and eight with particles in both neighbouring layers, corresponding to a coordination number $\mathcal{C}=12$. This structure will not change in the simulations described below (except for the results with polydispersity), i.e., the case of small amplitude perturbations is considered. Note that the packing is translationally invariant in the $x$ - and $y$-directions, but different in the $z$-direction, hence it is an anisotropic system (see Fig. 1). Polydisperse packings are obtained by using the regular structure where a tiny size distribution is given to the particles. The volume is kept constant, the deformations are thus tiny also so that the polydisperse packings are still ordered. 


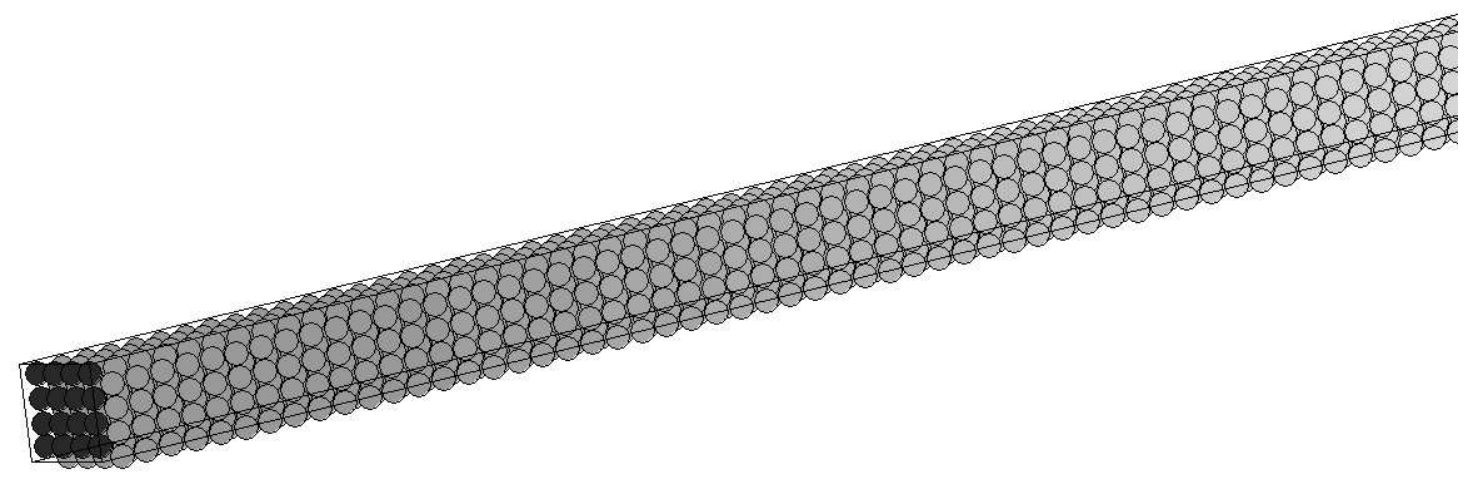

Figure 1. Snapshot of a part of the typical system used (long FCC packing). The dark particles belong to the fixed layer.

The packing is treated as piece of a larger sample via periodic boundaries, i.e., if a particle exits the simulation volume at one side, it enters at the opposite side at a corresponding position with the same velocity; particles feel each other across the periodic boundaries. While creating the regular structure, the position of particles is chosen such that the overlap is the same at all contacts in the system, hence giving an anisotropic stress, $\sigma_{x x}^{0}=\sigma_{y y}^{0} \neq \sigma_{z z}^{0}$ due to the anisotropic structure. The contact overlaps are chosen much smaller than the particle diameter, $\delta / d \approx 10^{-3}$. Before a small amplitude sound wave is excited (2), the system must be relaxed (1) first to a reasonable static equilibrium state.

(1) One possible criterion for a relaxed static state is the ratio of kinetic to potential energy. When this ratio becomes smaller than a given limit $\left(10^{-7}\right.$ in this study), the packing is said to be in a static state. For the regular, homogeneous packings used in the following, the system can be prepared immediately in a static configuration, whereas for the polydisperse packing (last paragraph) the system has to be relaxed. Note that such a relaxation typically takes much longer than a typical wave propagation simulation.

(2) Waves are excited by applying a small perturbation at one side of the system, i.e., by shifting a layer of particles. The wave vector, $\boldsymbol{k}$, and thus the wave propagation direction are perpendicular to the excited layer. Compressive $(\mathrm{P})$ and shear $(\mathrm{S})$ modes can be triggered by directing the perturbation parallel or perpendicular to the wave propagation direction, respectively. The typical packing used is long in the $z$-direction (200l $l_{0}$ with $l_{0}$ the distance between two layers), which allows to study the wave for a long time and large distances. We checked that the results do not depend on the extension in $x$ - and $y$-direction by comparing simulations with different size $l_{x}, l_{y}$. Therefore $l_{x}=l_{y}=4 d$ was used in order to reduce the number of particles. Such a long but thin system contains only $N=3200$ particles with radius $a=d / 2=0.001 \mathrm{~m}$. The mass of a spherical particle is $m=\rho_{0}(4 / 3) \pi a^{3}$, with the material density $\rho_{0}=2.10^{3} \mathrm{~kg} \mathrm{~m}^{-3}$. The total mass of the system is thus $M \approx 0.027 \mathrm{~kg}$. The stiffness material parameters in normal and tangential direction are $k=10^{5} \mathrm{~N} \mathrm{~m}^{-1}$ and $k_{t}$, given in units of $k$, e.g., $k_{t}=0.2 k$. Dissipation $\gamma_{t}=\gamma_{0}=0$ is used if not explicitly specified. This leads 
to a typical (two-particle) contact duration $t_{c}=2.03310^{-5} \mathrm{~s}$ and collision frequency $\omega_{c}=150 \mathrm{kHz}$, (the equations used to calculate these values are given in section 2.1). Note that the oscillation frequency of a particle in a crystal, with more than one contact, is higher than the collision frequency based on only one contact. For reliable numerical results, the criterion for the integration time-step is $\delta t_{\mathrm{MD}}<\frac{t_{c}}{50} \approx 4.10^{-7} \mathrm{~s}$.

\section{SIMULATION RESULTS}

In this section, a typical wave propagation simulation is presented. Then the effects of dissipation and friction are discussed. Also the wave propagation speed and the dispersion relations are determined and, finally, some irregularity is introduced in the crystal lattice.

\subsection{A typical wave propagation simulation}

As result of the strain-controlled perturbation of a layer, as described above in section 2.2, a plane compressive stress pulse ( $\mathrm{P}$ wave) is created and propagates in the system in $z$ direction, see Fig. 2. More specific, a $x, y$-layer is shifted by $\Delta z / d=10^{-4}$. This displacement amplitude, $\Delta z$, that excites the wave is still small as compared to the typical overlap $\Delta z / \delta=10^{-1}$. The travelling plane wave can be observed in various quantities like stress, displacement, kinetic energy, etc. Since the system is made of layers, it is possible to "record" the pulse at each layer as a function of time. Fig. 2 shows the scaled normal stress versus time at different positions along the wave propagation direction.

The advantage of periodic boundaries is that all particles are embedded in the same environment. As consequence, two opposite ends of the system are connected, and a tensile wave would travel in the direction opposite to the compressive pulse. The two waves will interfere after having travelled half of the system. In order to avoid this, and to maximise the distance that can be traveled by a pulse, two layers of particles at the opposite ends of the system ( $z$-direction) are fixed - the other two directions remain periodic. This avoids the tensile pulse, but not boundary reflections that lead to an oscillating "coda" traveling after the primary pulse, see Fig. 2. The alternative way of driving by specifying an initial velocity as perturbation of the layer (without controlling its displacement further on) excites a wave of the same nature and with the same properties, but a weaker primary pulse relative to the coda is observed.

With increasing distance from the exciting "source" layer the particles experience an increase in stress with a time delay and also with smaller amplitude and a slower rate of change, see Fig. 2. When the wave arrives at the other side, it is reflected from this "receiver" layer and travels back and forth between source and receiver several times, ever decreasing in amplitude (data not shown here). Modulations of the stress, much smaller than the agitation peak-stress, persist throughout the whole simulation. The analysis presented in the following considers only the initial, undisturbed wave, since 
interference with the reflected wave would disturb the signal. The shape of the signals and the wave speed will be examined next.

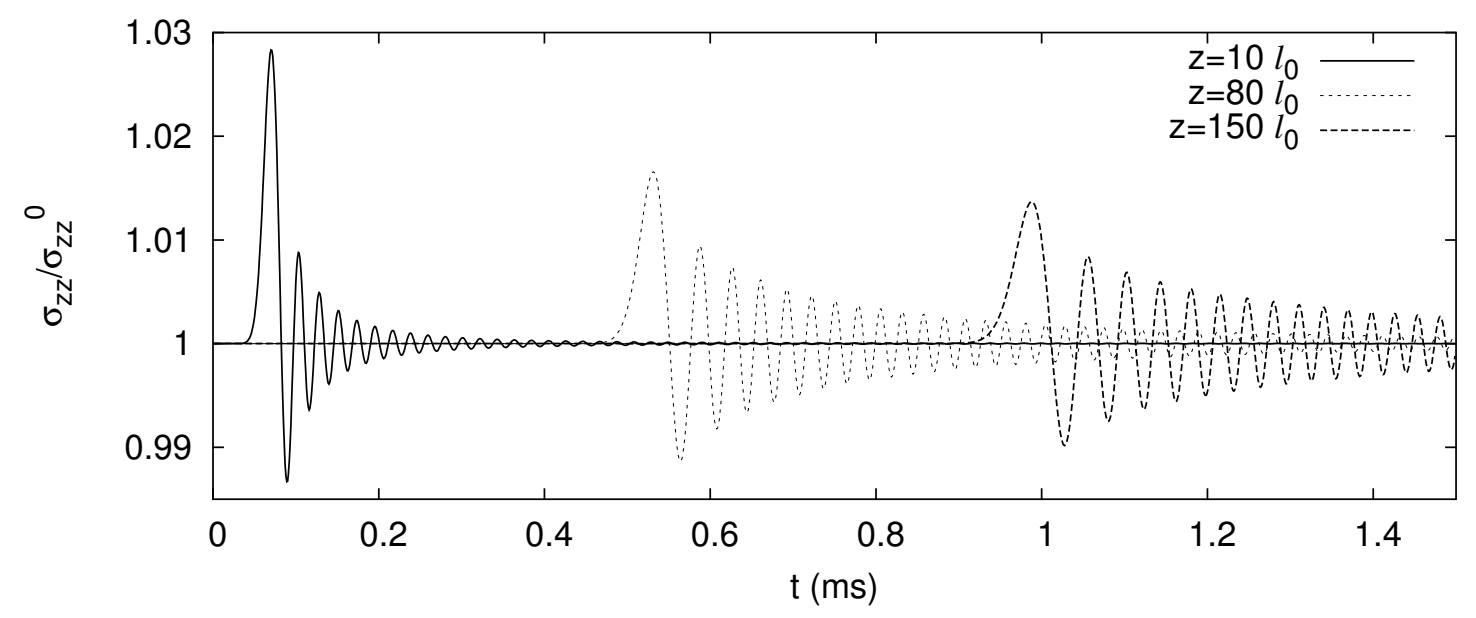

Figure 2. Normal stress $\left(\sigma_{z z}\right)$ scaled by the equilibrium stress $\left(\sigma_{z z}^{0}\right)$ as function of time at different positions $z / l_{0}=10,80$, and 150 , with the distance from the source, $z$, and the layer distance $l_{0}$.

\subsection{Signal shape and damping}

The pulse in Fig. 2 consists of two parts. The first strong peak corresponds to the increase of stress when the wave front arrives at the recording position. The second part, the "coda" of the signal, consists of secondary wave fronts created by the oscillation of the layer closest to the shifted layer (that stays fixed after the shift). During propagation, the first peak's amplitude decreases and its width increases. Note that there is no active dissipation, so that the described signal behaviour is caused by the frequency-dependent nature of the wave (dispersion), and the particular boundary condition.

When two real particles interact or collide with each other, a part of the kinetic energy is transformed into thermal energy, due to the local plastic deformation at the contact. It is not clear whether this phenomenon can be described properly by a linear spring-dashpot model. However, it appeared relevant for the sake of completeness, to study the influence of the dissipation model parameter on our results. More advanced contact models will be studied elsewhere [14].

As result of dissipation in the contact law (setting $\gamma_{0}>0$ and $r<1$, where $\gamma_{0}$ and $r$ were defined in section 2.1), both the amplitude of the first wave front and of the coda decrease, see Fig. 3 (Left). Note that the first pulse is affected much less than the coda, i.e., for a strong enough dissipation $\gamma_{0}>0.1 \mathrm{~kg} \mathrm{~s}^{-1}$, the coda has almost disappeard after ten layers, while the first peak continues travelling.

Fig. 3 (Right) shows the dependence of the peak amplitude evolution on dissipation. The strongest decay rate is observed when the dissipation time-scale $t_{\gamma}=m_{i j} / \gamma_{0}$ and a typical contact duration time-scale (oscillation period) become comparable: $t_{\gamma} \approx 2 t_{c}$. 
In summary, the wave is not affected by very weak dissipation. Stronger dissipation, however, lets the coda vanish but hardly changes the wave speed of the first peak, as discussed in the following paragraph.
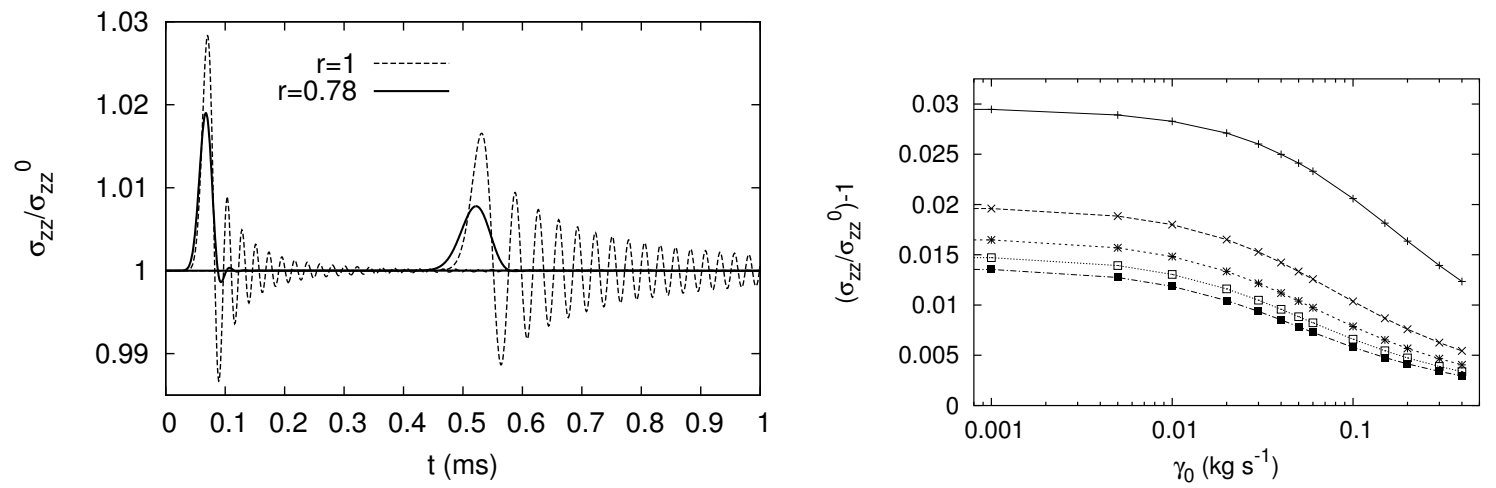

Figure 3. (Left) Two wave signals are plotted at $z / l_{0}=10$ and 80 , as in Fig. 2 , without damping (dashed line) and with damping (solid line). The dissipation strength is $\gamma_{0}=0.1 \mathrm{~kg} \mathrm{~s}^{-1}$, which corresponds to a restitution coefficient $r=0.78$ and a contact duration $t_{c}=2.03910^{-5} \mathrm{~s}$, comparable to the viscous damping time scale $t_{\gamma}=m_{i j} / \gamma_{0}=4.1910^{-5} \mathrm{~s}$.

(Right) The first peak amplitude at different positions $\left(z / l_{0}=10,45,80,115\right.$, and 150 from top to bottom) is plotted against the dissipation strength, displaying the decay of the wave amplitude with increasing dissipation and traveling time. The strongest decay rate is the point with the largest (negative) slope.

\subsection{The wave speed in frictionless packings}

One way to define the wave speed is to measure the time it takes the peak of the first pulse to travel a certain distance. Plotting the $z$-position of the peak against the time $t$ when it reaches that position (not shown here) gives an almost straight line. The slope of this line then gives the speed $V_{p}$ during propagation, see Fig. 4. Just assuming a constant speed (a linear fit to the $z$, $t$-data) leads to $V_{p 1} \approx 216 \mathrm{~m} / \mathrm{s}$. This disregards the interesting acceleration of the pulse early during propagation. In Fig. 4, the wave speed is compared to the simply fitted $V_{p 1}$ and to the theoretical prediction $V_{p z}$ as obtained from a micro-macro transition next. Note that in the article by Somfai et al [6], such an acceleration is also observed.

In order to compare the measured propagation speed with existing theories, it is assumed that the granular material behaves like an elastic continuum [7, 15]. The anisotropic relation between stress- and strain-increments involves a material tensor $\boldsymbol{C}$ of rank four. In symbolic and index notation (Einstein convention with summation over double indices) this reads in incremental form:

$$
\dot{\boldsymbol{\sigma}}=\boldsymbol{C}: \dot{\boldsymbol{e}}, \quad \text { or } \quad \dot{\sigma}_{i j}=C_{i j k l} \dot{e}_{k l},
$$

with the stress- and strain-rates on the left and right, respectively. This describes the response of our packing to large wavelength perturbations and implies the assumption of 


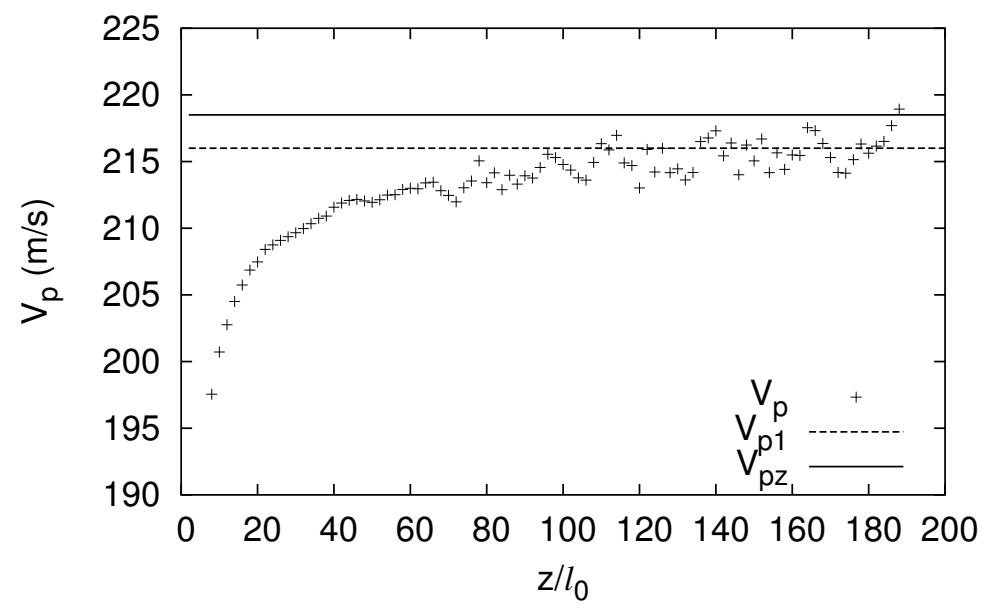

Figure 4. From the simulation without dissipation, see Fig. 3, the speed of the first peak maximum, $V_{p}$, is plotted as a function of the distance from the source. (A centerweighted average over five layers is used here. Also a higher output frequency was needed to obtain reliable data.) The dashed line indicates the average speed, $V_{p 1}$, and the solid line is the theoretical prediction, $V_{p z}$.

a constant, time-invariant material tensor, which can hold only for small deformations, and does not allow for opening or closing of contacts or even large scale rearrangements. In our regular system, neither of these conditions is violated.

The stiffness tensor of the material can be derived from the potential energy density via virtual displacement, see Refs. $[12,16]$,

$$
C_{\alpha \beta \gamma \phi}=\frac{1}{V} \sum_{p \in V}\left(k \sum_{c=1}^{C}\left(l^{2} / 2\right) n_{\alpha}^{c} n_{\beta}^{c} n_{\gamma}^{c} n_{\phi}^{c}+k_{t} \sum_{c=1}^{C}\left(l^{2} / 2\right) n_{\alpha}^{c} t_{\beta}^{c} n_{\gamma}^{c} t_{\phi}^{c}\right),
$$

with the branch vector $l \approx d$ and a representative volume $V$, e.g., the volume of the unit cell $V_{u}$. For a frictionless packing the second term involving $k_{t}$ is of course disregarded. From continuum theory, the $\mathrm{P}$ wave speed in $z$-direction is expected to be $V_{p z}{ }^{2}=C_{z z z z} / \rho$ and, e.g., the $\mathrm{S}$ wave speed in the same direction is $V_{s z}{ }^{2}=C_{z x z x} / \rho$. In Ref. [17] the prediction for the ratio $\left(V_{p z} / V_{s z}\right)^{2} \approx C_{z z z z} / C_{z x z x}=2$ was confirmed.

Inserting the contact normal and tangential vectors into Eq. (10), one can compute, e.g., $C_{z z z z}$, which leads to $V_{p z}=218.5 \mathrm{~m} / \mathrm{s}$ close to the linear fit estimate $V_{p 1} \approx 216 \mathrm{~m} / \mathrm{s}$ (see above section 3.3). This holds also for the plane S-wave propagating in the same direction (either polarised in the $x$ or $y$ direction), for which the theory gives $V_{s z}=154.5 \mathrm{~m} / \mathrm{s}$ and the linear fit $V_{s 1} \approx 153 \mathrm{~m} / \mathrm{s}$.

We performed a set of simulations exploring the anisotropy in the frictionless packing with $\mathrm{P}$ - and $\mathrm{S}$-waves in other directions for the propagation and for the particle movements and obtained similar agreement concerning the wave speeds. The anisotropy is summarised by the ratios of coefficients of the material tensor, $C_{x x x x} / C_{z z z z}=1.25$, $C_{z x z x} / C_{y x y x}=2$ and $C_{z z z z} / C_{z x z x}=2$. 


\subsection{Dispersion relation for frictionless packings}

The frequency dependence of the waves in our system can be studied by performing a Fourier analysis in time and space. From a $(t, x)$ data set in a system of length $l_{z}=200 l_{0}$, with spacing $\Delta z=l_{0}=d / \sqrt{2}$, every layer position is taken into account, i.e., 200 points in $x$-space are available. The time-window $0 \leq t \leq t_{\max }$ (with time step $\Delta t=10^{-6}$ s and $t_{\max }=1199 \Delta t$, i.e., 1200 data points), is chosen such that the wave has not yet arrived at the end of the system, so that reflections are not included in the signal.
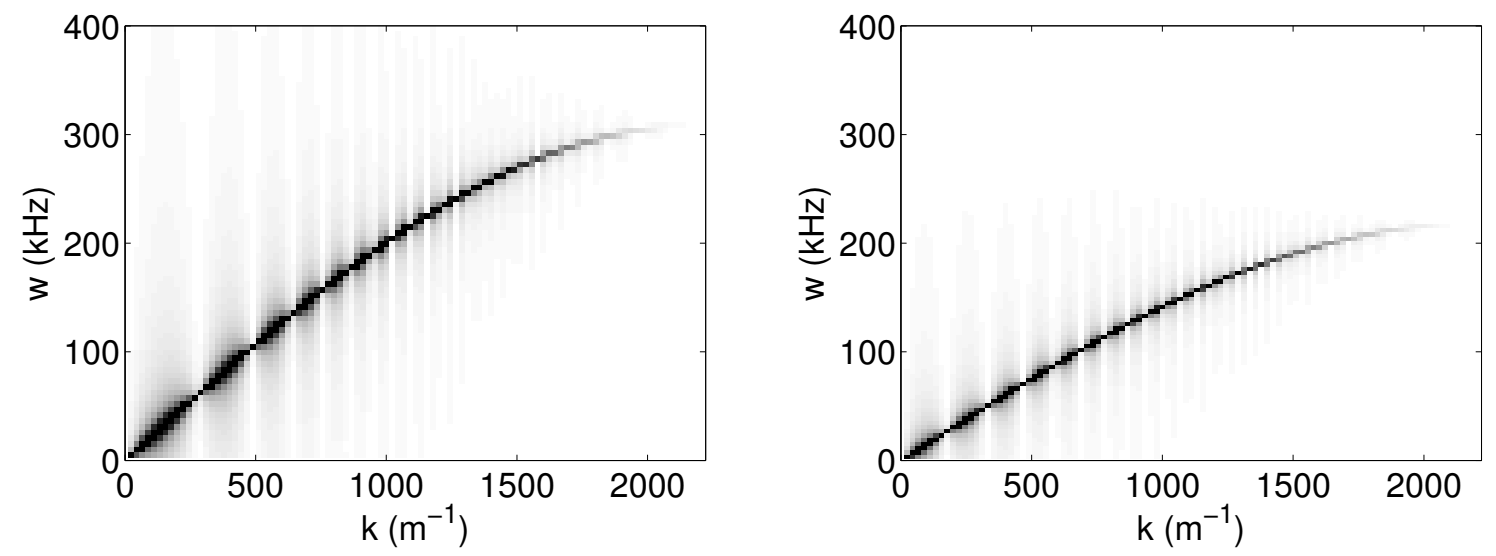

Figure 5. Dispersion relations (grey-scale corresponds to the amplitude, absolute value, of the Fourier coefficients) for $\mathrm{P}$ wave (Left) and $\mathrm{S}$ wave (Right) propagating in $z$ direction in the anisotropic packing.

The dispersion relations are obtained for $\mathrm{P}$ and $\mathrm{S}$ waves propagating in $z$ direction, see Fig. 5. The MATLAB function fft2 has been used, which returns the Fourier coefficients in an indexed $(\omega, k)$-field $F(\omega, k)$ of the same size as the input $(t, x)$ data. Transformation to frequency and wave-number is performed by multiplying the index with $\Delta \omega=2 \pi / t_{\max }$ and $\Delta k=2 \pi / l_{z}$. Special care has to be taken that the indices corresponding to $\omega=0$ and $k=0$ are properly shifted to zero.

In order to obtain a sine fit $\omega(k)=\omega_{0} \sin \left(\frac{k l_{0}}{2}\right)$ to the Fourier data, the locations of the maximal Fourier coefficients on the frequency axis are determined by a powerlaw weighted average, $\omega_{\max }(k)=\Sigma F(\omega, k)^{\phi} \omega / \Sigma F(\omega, k)^{\phi}$, with $\phi=4$. The quality of the fit is impressive with respect to both shape of the curve and magnitude of $\omega_{0}$. The sine function is consistent with the theoretical work by Suiker et al. [4], where the dispersion relation for $2 \mathrm{D}$ lattices is discussed. According to the fits, the maximal frequencies are $\omega_{0}^{p z}=309132 \mathrm{~s}^{-1}, \omega_{0}^{s z}=218437 \mathrm{~s}^{-1}$, and the minimal wavelength is $2 l_{0}$. The largest wave speed is obtained for $V_{p z}^{\omega}(k=0)=\left.\frac{d \omega}{d k}\right|_{(k=0)}=\omega_{0} l_{0} / 2=218.59 \mathrm{~m} / \mathrm{s}$, and $V_{s z}^{\omega}(k=0)=154.45 \mathrm{~m} / \mathrm{s}$. These values have to be compared to the prediction based on the micro-macro transition: $V_{p z}=218.50 \mathrm{~m} / \mathrm{s}$ and $V_{s z}=154.50 \mathrm{~m} / \mathrm{s}$, respectively, in order to appreciate the perfect agreement between an effective continuum theory and the discrete simulation. 
The acceleration of the wave observed above can now be quantitatively related to an increasing wave-length during propagation. As crude approximation, the duration $T$ of the first oscillation of a signal (based on first-arrival time - one per-cent peak level), see Fig. 2, at a given position ( $z$ ), multiplied by the corresponding speed $V$, see Fig. 4 , gives an estimated wavelength $\lambda=V T$, for this part of the signal at the given position. We obtain $\lambda / l_{0} \approx 8,9,10,16$, and 19 , with error-margin \pm 1 , at $z / l_{0}=10,15,20,80$, and 150 , respectively.

Using the dispersion relation, a typical wave-number can be related to a local wave propagation speed. In subsection 3.3 , the wave is excited with a minimal wave-length and rapidly adapts to a speed of $V_{p z}^{\omega}\left(z / l_{0}\right) \approx 208 \mathrm{~m} / \mathrm{s}$ at $z / l_{0} \approx 20$, and accelerates further on, see Fig. 4. The dispersion relation leads to a prediction of the wave-number as function of the local wave-speed at position $z / l_{0}$,

$$
k\left(\frac{z}{l_{0}}\right)=\frac{2}{l_{0}} \arccos \frac{V_{p z}^{\omega}\left(z / l_{0}\right)}{V_{p z}^{\omega}(k=0)},
$$

an expression that works well for short and intermediate distances. At different positions one finds the wavelengths $\lambda=2 \pi / k$ with $\lambda_{10} \approx 8.3 l_{0}, \lambda_{15} \approx 9.2 l_{0}, \lambda_{20} \approx 10.1 l_{0}$, $\lambda_{80} \approx 13.9 l_{0}, \lambda_{150} \approx 17.0 l_{0}$, consistent with the crude average obtained from the oscillation duration of the signal for the shorter distances. At longer distances from the source, the strong fluctuations and the fact that the slope of the dispersion relation only weakly changes for small $k$, leave the prediction with too large error margin to be useful.

The typical wavelength is increasing while the wave (slightly) accelerates. The dispersion relation acts such that low-frequency/long wavelength components are faster than the high-frequency wave components with smaller wavelength. With other words, narrow pulses can not travel fast, and high frequency perturbations $\left(\omega \approx \omega_{0}\right)$ will practically not propagate at all, so that the larger wavelenghts remain travelling after long distances.

This detailed quantitative study of the classical dispersion relation in a crystal (see literature in solid state physics) is only the reference basis for more detailed research on sound propagation in polydisperse, disordered, frictional and anisotropic granular media.

\subsection{The influence of friction}

Friction is now introduced in the same system as described previously. The new parameters are $\mu$, the friction coefficient, which is kept constant to $\mu=0.5$ and $k_{t}$ the tangential stiffness for which we performed a parameter study with the following values: $k_{t} / k=0,0.2,0.5,1,1.5$ and 2 . Since there are no sliding contacts, due to the small amplitude perturbation, neither are differences of static and dynamic friction coefficients, or different magnitudes of $\mu$, relevant here (data not shown). Of course by choosing an artificially low value for $\mu(\mu=0.001)$, see [17], sliding contacts occur and 
influence the results. This was checked by performing several simulations with $\mu_{d} \neq \mu_{s}$ and different magnitudes. Since details of the friction model are not relevant here, we use $\mu_{d}=\mu_{s}=\mu=0.5$ in the following. The system is prepared without friction such that $f_{t}=0\left(f_{t}\right.$ is the contact force in the tangential direction) at $t=0$ and $f_{t} \neq 0$ as soon as displacements and deformations occur. Note that a different preparation procedure (data not shown) will lead to a different initial condition, with contacts close to the sliding limit, and thus to different observations and conclusions.

The same analysis is performed as before (without friction) for both $\mathrm{P}$ - and S-waves. In both cases ( $\mathrm{P}$ - and $\mathrm{S}$-wave) the first peak amplitude decreases as the tangential stiffness $\left(k_{t}\right)$ is increasing. Also the "coda" tends to decay stronger and vanishes. The velocity increases with $k_{t}$ increasing and it is pretty well predicted for the $\mathrm{P}$-wave by using Eq.(10) for the stiffness tensor and using the simulation data in order to get the direction of both contact normals and tangential springs, see Fig. 6 (theory). However for the S-wave it is not possible to use the same formula for the stiffness tensor, since the influence of rotations is strong and irregular in the tangential direction of contacts and renders Eq. (10) inappropriate. A more complete model like introduced in [4, 5] is needed in order to take rotations into account.

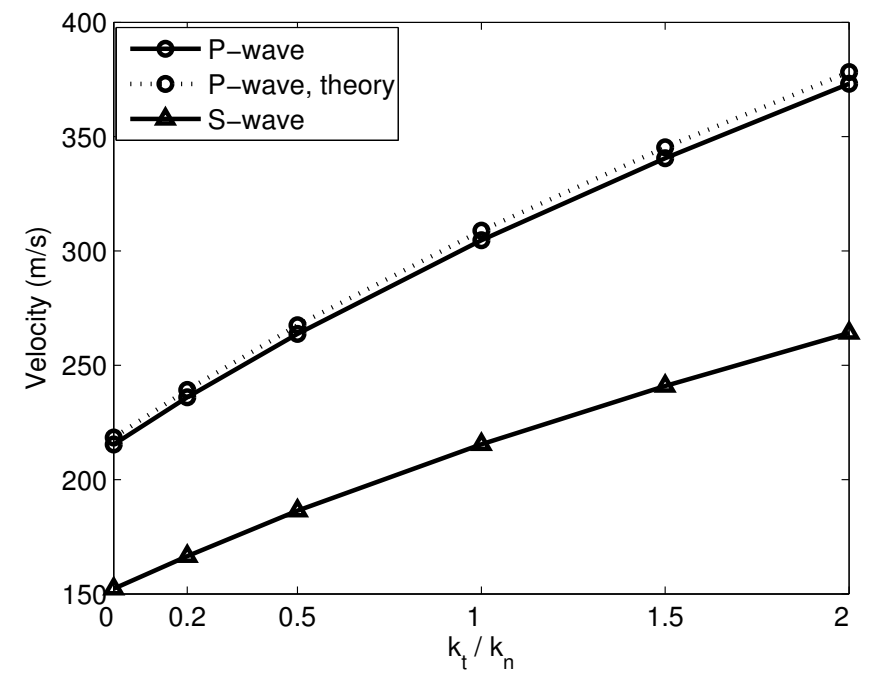

Figure 6. Wave velocities as function of the ratio $k_{t} / k_{n}$, with the normal stiffness, $k_{n}=k$, as introduced in section 2 (simulation and theory as described in the text).

The same agreement as in section 3.4, between theory and simulations, is obtained for the dispersion relation for the $\mathrm{P}$-wave with friction. For the S-wave, a new branch appears in the $\omega(k)$ picture for negative wave numbers, see Fig. 7 , and the original branch deviates from the sine shape towards higher frequencies and higher wave velocities. Larger wave numbers (smaller wavelength) modes can propagate with higher speed (slope of the dispersion relation), dependent on the magnitude of $k_{t}$. The frequency range of the new branch is tuneable, i.e., smaller $k_{t}$ leads to 
smaller frequencies. The positive trend of this branch indicates that it is related to a wave travelling in the same direction as the S-wave. However, we still have no clear interpretation for this branch. Presumably it is a trace of the rotational degree of freedom of the particles. A more quantitative study of wave propagation in frictional, rotating systems will be presented elsewhere [14].

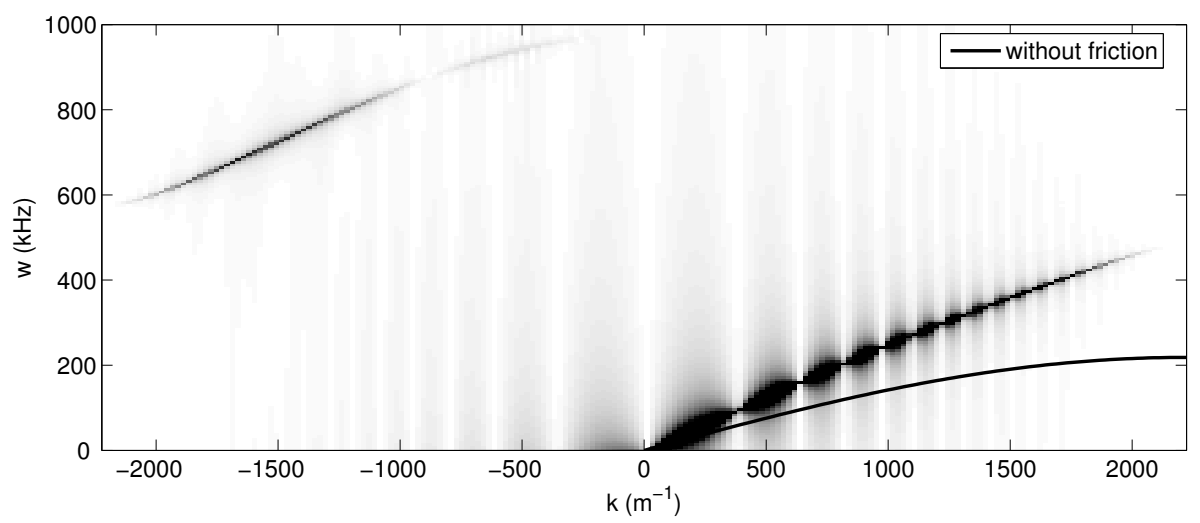

Figure 7. Dispersion relation (grey-scale corresponds to the amplitude of the Fourier coefficients) for the S-wave with $k_{t} / k_{n}=2$. The solid line gives the best fit to the $\mathrm{P}$-wave dispersion relation from the system without friction, as discussed in section 3.4 .

\subsection{Frictionless, slightly polydisperse and ordered systems}

Some polydispersity is now introduced in the crystal structure, by applying a size distribution to the particles. The distribution is homogeneous, centered at the original monodisperse value of the radius $(a=0.001 \mathrm{~m})$ and has a width $(2 \Delta a)$ of the order of the overlap $\left(\delta / a=10^{-3}\right)$. More precisely we studied three different cases with $\Delta a=\frac{\delta}{2}, \delta$ and $2 \delta$. The simulations are performed with the same parameters as in the ordered, elastic case (section 3.1). The difference is that a preparation-simulation is needed in order to relax the system, as mentioned in section 2.2. As one can see from Fig. 8, the signal is strongly affected for $\Delta a=2 \delta$. For $\Delta a=\delta / 2$, the signal is practically unchanged, and for $\Delta a=\delta$ we obtained something in between the two other cases (data not shown). In the case of $\Delta a=2 \delta$, the size variation is $0.2 \%$ of the radius but $200 \%$ if related to the overlap $(\delta)$.

The fact that polydispersity has to be related to the overlap rather than the particle size was already reported in [10]. As consequence, strong non-linear effects at the contacts, such as opening and closing, are evidenced. Note that the coordination number of the relaxed, disordered packing $(\Delta a=2 \delta)$ is $C \simeq 9.975$. This represents a loss of $17 \%$ of the contacts as compared to the ordered system $(C=12)$. During the wave propagation the coordination number is oscillating (as result of opening and closing of contacts), by less than $0.01 \%$. In this non-linear context, the linear theory used to 


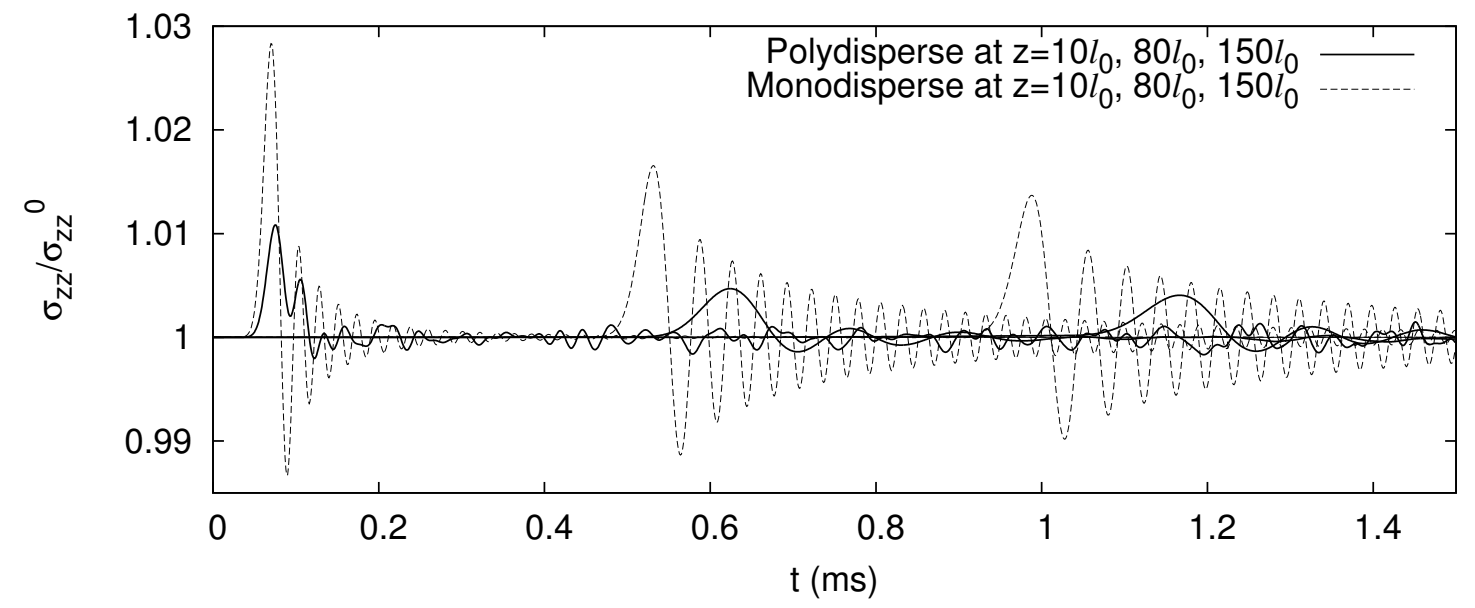

Figure 8. Normal stress $\left(\sigma_{z z}\right)$ scaled by the equilibrium stress $\left(\sigma_{z z}^{0}\right)$ as function of time at different positions $z / l_{0}=10,80$, and 150 , with the distance from the source, $z$, and the layer distance $l_{0}$. Comparison between the monodisperse system (see Fig. 2) and the polydisperse system with $\Delta a=2 \delta$.
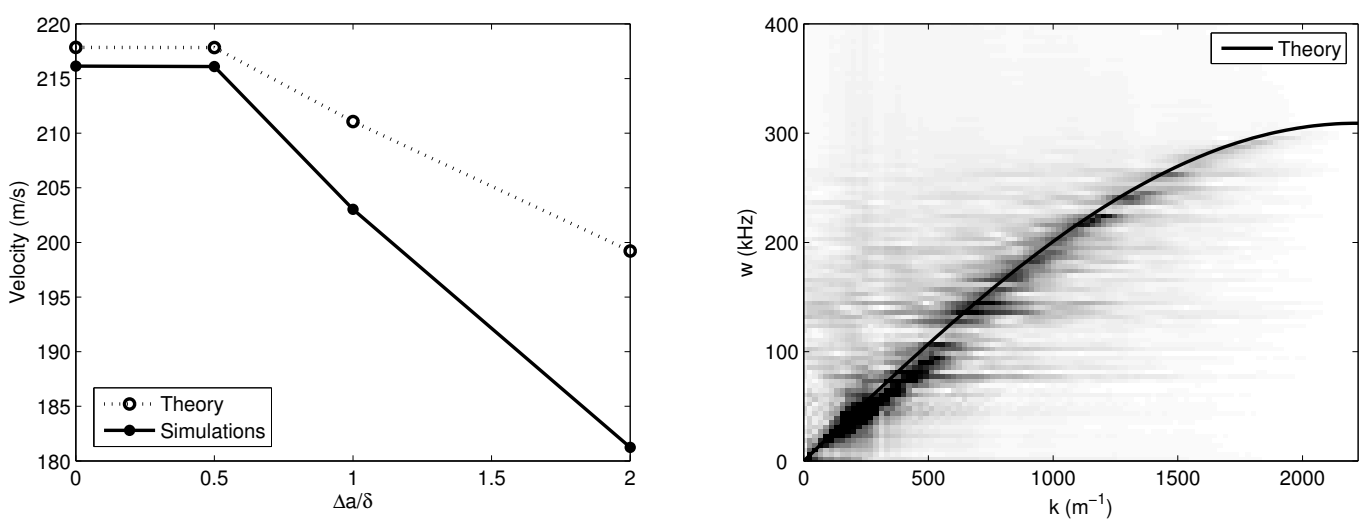

Figure 9. (Left) P-wave velocities as function of polydispersity (simulations and theory). (Right) Dispersion relation (grey-scale corresponds to the amplitude of the Fourier coefficients) for the P-wave in the polydisperse system, $\Delta r=2 \delta$. The solid line corresponds to the dispersion relation from the monodisperse, ordered system from section 3.4.

predict the wave velocity fails (discrepancies up to $9 \%$ for $\Delta a=2 \delta$ ). For a theoretical appraoch on the moduli, that considers random arrays, see Ref. [13]. Also the dispersion relation is much broader, more noisy, and random gaps seem to appear, although the sine shape is still visible (see Fig. 9).

\section{CONCLUSION}

Wave propagation was examined in three dimensional regular (crystal) monodisperse packings of spheres, for compressive (P) and shear (S) propagation modes. Different 
dissipation strength and friction coefficients were used and an interesting acceleration of the wave during propagation was observed for both $\mathrm{P}$ - and $\mathrm{S}$-waves in frictionless systems.

For the wave speeds, quantitative agreement was obtained between simulations and theoretical predictions based on a micro-macro computation of the stiffness material tensor for the anisotropic lattice. Also the dispersion relation agrees perfectly well with theory and the observed acceleration of the travelling wave can be related to the dispersion and widening of the pulse: the initially narrow pulse travels slower than the wider, more developed pulse. Close to the source, the major wave number (wave length) can be well predicted as function of the wave speed.

The study of frictional packings indicates the importance of rotations, and also the limitations of the proposed theory for the wave speed. In the dispersion relation, the S-wave branch becomes steeper and more straight (the sine-shape is lost), and a second branch at higher frequencies occurs.

Finally in the last paragraph, weak polydispersity was introduced and more generally, non-linearity effects are evidenced already for rather small disorder. The dispersion relation becomes more noisy but the shape is maintained.

All this shows that by capturing already a certain amount of relevant and interesting features of wave propagation in granular materials, the model proposed seems to be an appropriate starting point in order to investigate quantitatively and more deeply the phenomenon of wave propagation in polydisperse, inhomogeneous, anisotropic, dense, frictional granular materials.

\section{ACKNOWLEDGEMENTS}

Helpful discussions with G. Herman and A. Suiker are appreciated. This work is part of the research programme of the Stichting voor Fundamenteel Onderzoek der Materie (FOM), financially supported by the Nederlandse Organisatie voor Wetenschappelijk Onderzoek (NWO) and the Stichting Shell Research.

\section{References}

[1] C.-h. Liu and S. R. Nagel. Sound in sand. Phys. Rev. Lett., 68(15):2301-2304, 1992.

[2] X. Jia, C. Caroli, and B. Velicky. Ultrasound propagation in externally stressed granular media. Physical Review Letters, 82(9):1863-1866, 1999.

[3] R. Garcia-Rojo, H. J. Herrmann, and S. McNamara, editors. Powders and Grains 2005, Leiden, 2005. A. A. Balkema Publishers.

[4] A. S. J. Suiker, A. V. Metrikine, and R. de Borst. Comparions of wave propagation characterisitcs of the Cosserat continuum model and corresponding discrete lattice models. Int. J. of Solids and Structures, 38:1563-1583, 2001.

[5] A. S. J. Suiker, and R. de Borst. Enhanced continua and discrete lattices for modelling granular assemblies. Phil. Trans. R. Soc. A, 363:2543-2580, 2005.

[6] E. Somfai, J-N. Roux, J. H. Snoeijer, M. van Hecke, and W. van Saarloos. Elastic wave propagation in confined granular systems. Physical Review E, 72(2):021301, 2005. 
[7] A. L. Fetter and J. D. Walecka. Theoretical Mechanics of Particles and Continua. Dover Publisher, 2003.

[8] P. A. Cundall and O. D. L. Strack. A discrete numerical model for granular assemblies. Géotechnique, 29(1):47-65, 1979.

[9] L. Brendel and S. Dippel. Lasting contacts in molecular dynamics simulations. In H. J. Herrmann, J.-P. Hovi, and S. Luding, editors, Physics of Dry Granular Media, page 313, Dordrecht, 1998. Kluwer Academic Publishers.

[10] S. Luding. Stress distribution in static two-dimensional granular model media in the absence of friction. Phys. Rev. E, 55:4720-4729, 1997.

[11] S. Luding. Micro-macro transition for anisotropic, frictional granular packings. Int. J. Sol. Struct., 41:5821-5836, 2004.

[12] S. Luding. Anisotropy in cohesive, frictional granular media. J. Phys. Condensed Matter, 17(24):S2623-S2640, 2005.

[13] J. T. Jenkins et al. Fluctuations and the effective moduli of an isotropic, random aggregate of identical, frictionless spheres. Journal of the Mechanics and Physics of Solids, 53(1):197-225, 2004.

[14] O. Mouraille and S. Luding. Dispersion relations for frictional, random granular media. in preparation.

[15] P. A. Vermeer, S. Diebels, W. Ehlers, H. J. Herrmann, S. Luding, and E. Ramm, editors. Continuous and Discontinuous Modelling of Cohesive Frictional Materials, Berlin, 2001. Springer. Lecture Notes in Physics 568.

[16] S. Luding. Granular media: Information propagation. Nature, 435:159-160, 2005.

[17] O. Mouraille and S. Luding. Sound propagation in dense, frictional granular materials. In R. Garcia-Rojo, H. J. Herrmann, and S. McNamara, editors, Powders and Grains 2005, page 319, Leiden, 2005. A. A. Balkema Publishers. 